\title{
A regional quality improvement project to improve the standards of care for people with diabetes who are on maintenance haemodialysis
}

\author{
Authors: Apexa Kuverji, ${ }^{A}$ June James, ${ }^{B}$ Robert Gregory, ${ }^{B}$ Andrew Frankel ${ }^{C}$ and James Burton ${ }^{A}$
}

Table 1. Standards of care for people with diabetes on maintenance haemodialysis

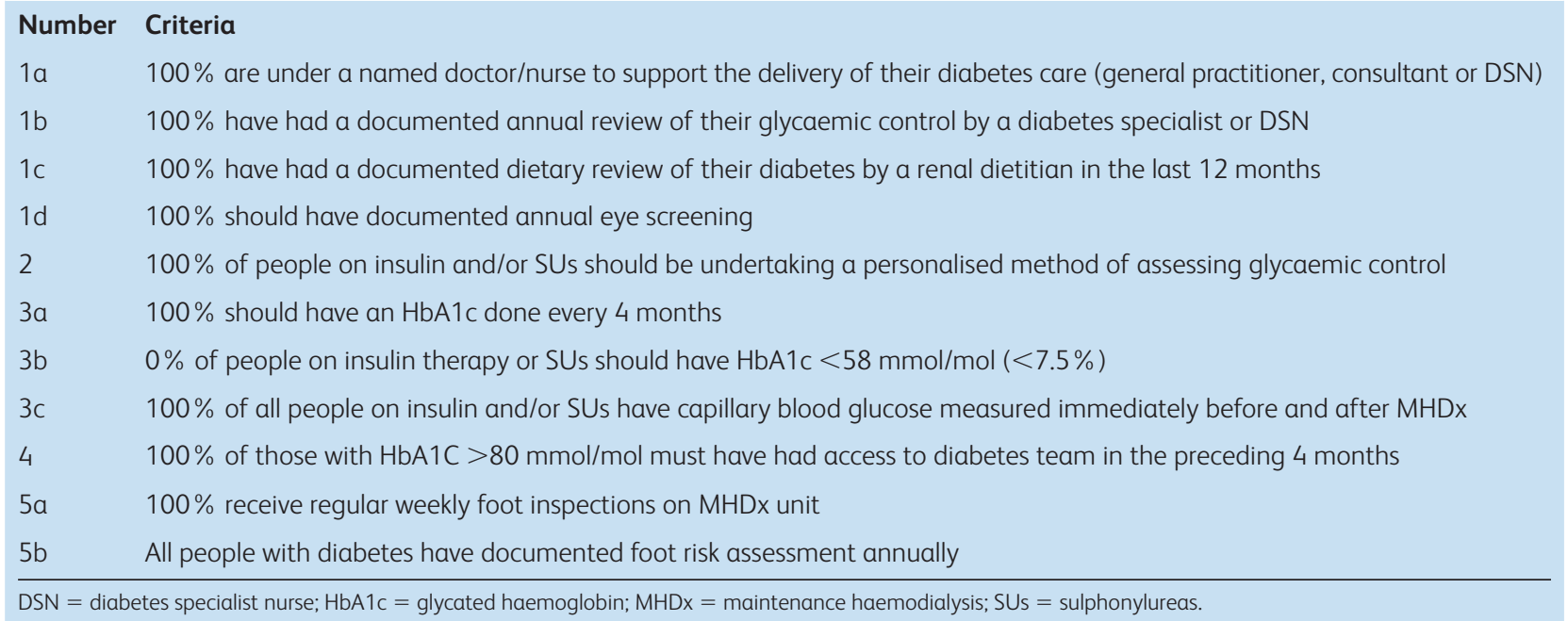

\section{Introduction}

People with end-stage renal disease (ESRD) caused by diabetes account for $29.4 \%$ of people on maintenance haemodialysis (MHDx). When taking into account those with diabetes not causing ESRD, this figure rises up to $40 \%$ in some units. ${ }^{1}$ People with diabetes and kidney disease are at a particularly high risk of complications such as non-traumatic lower extremity amputation, cardiovascular events and death, with overall survival on MHDx in people with diabetes being approximately half that of their non-diabetic peers (3.7 vs 7.0 years). ${ }^{2-4}$ In 2016, guidelines were published by the Joint British Diabetes Societies in conjunction with the Renal Association, aimed at defining good-quality care for a person with diabetes on MHDx. ${ }^{5}$ These guidelines have been disseminated widely but evidence is lacking in their use in the dayto-day management of patients. This quality improvement project (QIP) uses a multidisciplinary team (MDT) approach to develop a set of standards based on the national guidelines, identify areas

Authors: ${ }^{\text {A}}$ John Walls Renal Unit, Leicester, UK; ${ }^{B}$ Leicester Diabetes Centre, Leicester, UK; ' Diabetes Care in Haemodialysis Working Group, UK of substandard care and put interventions in place to improve this across the Leicester Renal Network.

\section{Materials and methods}

The standards highlighted in Table 1 apply to all people with diabetes on MHDx. Data were collected in May to August 2019 from a combination of paper medical records, IT systems and patient questionnaires.

\section{Results and discussion}

A total number of $151 \mathrm{MHDx}$ patients with diabetes were identified from three dialysis units. Baseline data identified differences in organisational care between the NHS hospital-based dialysis unit, NHS non-hospital-based (satellite) unit and non-NHS satellite unit (Fig 1).

The differences in certain standards (1a, 1c, 4, 5a and 5b) could be attributed to many factors, including:

> different dietitian referral pathways (routine review vs referralbased only)

$>$ different staffing contracts (NHS vs non-NHS)

$>$ geographical proximity to secondary care services. 


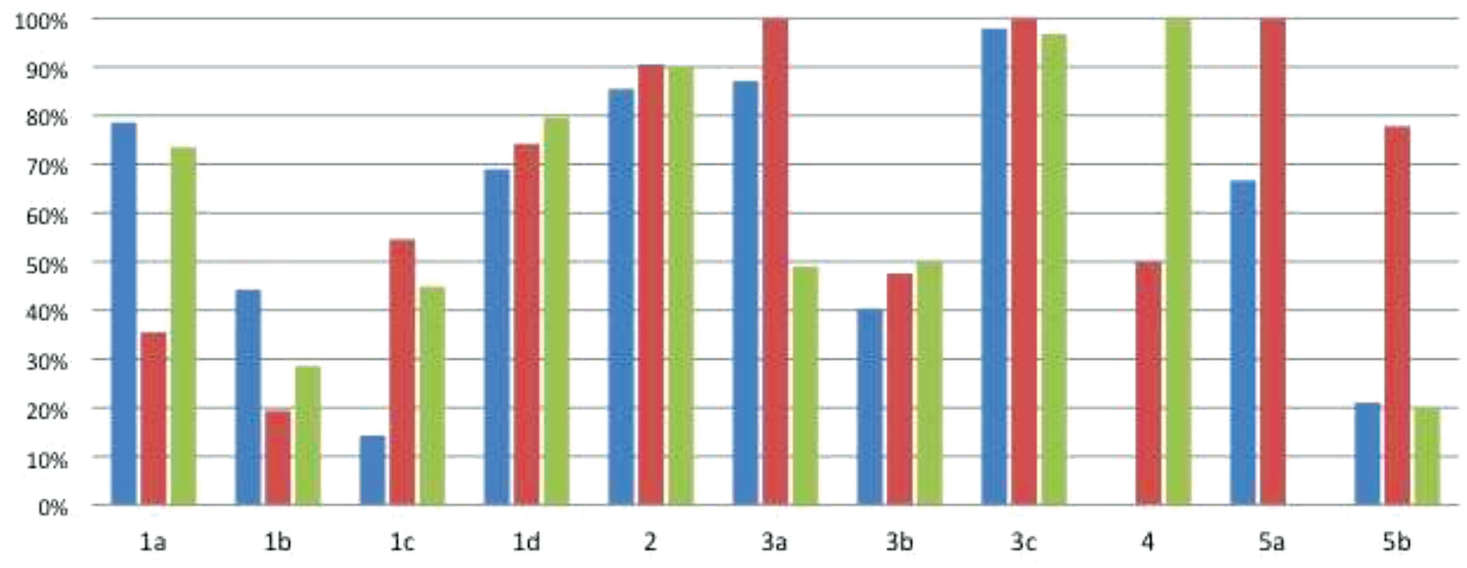

" NHS Hospital Unit (N=71) May $2019=$ NHS Non-hospital Unit (N=31) Aug $2019=$ Non-NHS satellite Unit (N=49) Aug 2019

Fig 1. Percentage of people achieving standards at three dialysis units in Leicestershire.

We identified patients who were not getting annual diabetic reviews, eye screening and foot checks. Similarly, patients at high risk of hypoglycaemia were recognised. Areas of good practice were also seen.

> NHS non-hospital-based unit achieved $100 \%$ compliance with standards $3 a, 3 c$ and $5 a$ by utilising a diabetes care plan inserted in each patient's dialysis folder.

There was a difference between patient perceived care (from patient questionnaire) and objective documented care given (medical records), with the discrepancy rate being up to $41.3 \%$ for some standards. This highlights the potential factors, such as memory/recall, clear communication and documentation that influence patient experience.

\section{Conclusion}

This QIP has identified areas where standards, based on national guidelines, are underachieved across different units and the barriers to achieving them. Interventions to implement change include:

$>$ presenting results to renal dietitians

> creating a virtual diabetes-renal MDT to discuss complex cases and bridge both aspects of care

> diabetes education days for dialysis nurses designed and delivered by ‘Diabetes Care in Haemodialysis' working group

$>$ creating and using a hypoglycaemia risk assessment tool.

These interventions are currently being implemented, with re-audit planned to assess any improvement in the care provided. Despite the barriers, there are areas of good practice, that when shared with other units, may benefit the wider dialysis population.

\section{Conflicts of interest}

None declared.

\section{References}

1 The Renal Association. UK Renal Registry 21st annual report: Data to 31/12/2017. Bristol: UK Renal Registry, 2019. www.renalreg. org/wp-content/uploads/2019/05/21st_UKRR_Annual_Report.pdf [Accessed 29 September 2019].

2 Eggers PW, Gohdes D, Pugh J. Nontraumatic lower extremity amputations in the Medicare end-stage renal disease population. Kidney International 1999;56:1524-33.

3 Palanca A, Castelblanco E, Betriu À et al. Subclinical atherosclerosis burden predicts cardiovascular events in individuals with diabetes and chronic kidney disease. Cardiovascular Diabetology 2019:18:93.

4 The Renal Association. UK Renal Registry 20th annual report. Bristol: UK Renal Registry, 2018. www.renalreg.org/wp-content/ uploads/2018/06/20th-Annual-Report_web_book.pdf [Accessed 29 September 2019].

5 Frankel A, Kazempour-Ardebili S, Bedi R et al. Management of adults with diabetes on the hemodialysis unit: summary of new guidance from the Joint British Diabetes Societies (JBDS) and the Renal Association. Br J Diabetes 2016;16:69e77. https://abcd. care/sites/abcd.care/files/resources/JBDS_RenalGuide_2016.pdf [Accessed 29 September 2019]. 